\title{
P. Frath, J. C. Herreras (coord.), Plurilinguisme et créativité scientifique
}

«Plurilinguisme », coll. dirigée par l'OEP, n² 2016/2, thebookedition.com, ISBN : 978-29-537-2991-7

\section{Asja Prohić}

\section{(2) OpenEdition}

\section{Journals}

Édition électronique

URL : http://journals.openedition.org/esp/2693

DOI : 10.4000/esp.2693

ISSN : 2532-0319

Éditeur

Centre d'Information sur l'Éducation Bilingue et Plurilingue

Édition imprimée

Date de publication : 1 juin 2018

Pagination : 105-109

ISSN : 1127-266X

Référence électronique

Asja Prohić, «P. Frath, J. C. Herreras (coord.), Plurilinguisme et créativité scientifique », Éducation et sociétés plurilingues [En ligne], 44 | 2018, mis en ligne le 08 février 2019, consulté le 24 septembre 2020. URL : http://journals.openedition.org/esp/2693 ; DOI : https://doi.org/10.4000/esp.2693 


\section{GOMPTE-RENDU d'OUVRAGE}

\section{P. Frath, J. C. Herreras (coord.), Plurilinguisme et créa- tivité scientifique, "Plurilinguisme», coll. dirigée par l'OEP, $\mathrm{n}^{\circ}$ 2016/2, thebookedition.com, ISBN: 978-29- 537-2991-7}

\section{Asja Prohić}

Parole chiave: Osservatorio europeo del Plurilinguismo, plurilinguismo, creatività scientifica

Key-words: European Observatory for Plurilingualism, multilingualism, scientific creativity

Oe volume publie les interventions à la journée d'étude organisée en 2013 par le «Pôle Recherche» de l'Observatoire Européen du Plurilinguisme (OEP) et l'Université Paris Diderot sur le thème du plurilinguisme et de la créativité scientifique.

Coordonné par P. Frath, Professeur émérite à l'Université de Reims Champagne-Ardenne et J. C. Herreras, Professeur des Universités en linguistique espagnole de l'Université Paris Diderot-Paris, tous deux membres du Comité de rédaction de la collection «Plurilinguisme» de l'OEP, l'ouvrage réunit dix articles rédigés pour certains par des chercheurs en sciences humaines et sociales et d'autres par des mathématiciens, physiciens, biologistes. Ce choix d'auteurs permet d'aborder le sujet de manière interdisciplinaire et d'enrichir le débat théorique par des témoignages de pratiques réelles.

Le ton est donné avec le premier texte signé par le mathématicien L. Lafforgue qui explique les ressorts de la causalité «choix du français/créativité scientifique» par des conditions psychologiques, morales et culturelles. Psychologiques, en adoptant une attitude combative et résistante; morales, signifiant que l'on accorde plus d'importance à la recherche en elle-même qu'à sa communication; culturelles, enfin, témoignant de l'attachement à et puisant dans les sources scientifiques de son propre pays.

J.-M. Lévy-Leblond s'attaque d'emblée, dans son texte, à l'idée qu'il y aurait un langage scientifique, véhicule neutre dénué de toute nuance, porteur de termes extrêmement formalisés. Oui, les 
Compte-rendu A. Prohić mathématiciens ou les chimistes produisent une terminologie spécifique, mais ils ne diffèrent pas en cela des autres corps de métier. L'auteur cherche également à dissiper l'illusion de l'intérêt d'une langue unique pour la science. L'analogie souvent faite avec le latin est, selon lui, totalement fallacieuse, notamment en ce qu'elle cache des enjeux de domination évidents alors que le latin médiéval n'a jamais été la langue d'une hyper-puissance mondiale, et sachant que c'est justement l'usage des langues populaires qui a permis le révolution scientifique de la fin du XVIIe siècle.

Sur l'exemple de la physique, l'auteur retrace l'histoire de la création terminologique en science, distinguant trois principales périodes, la dernière correspondant à l'apparition de la technoscience et, par manque quasi-général de formation culturelle des scientifiques, à une terminologie dominée par des enjeux économiques et médiatiques.

En conclusion, J.-M. Lévy-Leblond nous livre quelques exemples de réussite terminologique mais aussi des traductions malencontreuses et propose un travail explicite et délibéré de réflexion linguistique en science à partir de la diversité des langues existantes. La physique des particules est une science caractérisée par de très grandes collaborations internationales et un fort nomadisme des chercheurs que M. Zito décrit dans son texte, moitié témoignage de son propre parcours de scientifique, moitié réflexion sur les conséquences de l'hégémonie de l'anglais dans son domaine de recherche. Cette contribution a le mérite de nous introduire au cœur d'échanges entre scientifiques et les langues qu'ils y utilisent. On apprend, sans grande surprise, que l'anglais (ou le globish, comme il l'appelle) est prédominant dans ces échanges, qu'ils soient informels ou plus formels, mais que la moitié à peu près, d'entre eux, se déroule en français ou en d'autres langues, italien entre Italiens, grec entre Grecs, etc.

La principale conséquence néfaste induite par le processus d'homogénéisation linguistique ne se situe donc pas, pour l'auteur, dans la langue qui est utilisée entre pairs, mais dans l'effort de la vulgarisation que les scientifiques sont prêts à faire pour la société afin de ne pas s'en isoler. Le maintien du lien entre langue maternelle et univers scientifique est également, selon lui, seule source d'enrichissement intellectuel aussi bien pour le chercheur que pour son pays.

Sous le titre drolatique de «Biologie et/or Biology: Témoignage d'un schizophrène», $\mathrm{P}$. Regnier nous explique toutes les raisons pragmatiques pour lesquelles il est essentiel pour un chercheur de 
Compte-rendu A. Prohić langue française de maîtriser au mieux l'anglais de service, cela notamment pour pouvoir publier et communiquer ses résultats au plus grand nombre. L'auteur préconise, cependant, une politique d'enseignement du français et en français pour tous les étudiants, les enseignants et les chercheurs qui viennent en France.

La seule contribution proposée dans une autre langue que le français, l'allemand, est celle de R. Mocikat. L'auteur décrit l'hégémonie de l'anglais dans le domaine de la recherche et de l'enseignement universitaire en Allemagne. Par conséquent, l'allemand n'est plus utilisé en science, menant ainsi à une perte de terminologie et de créativité scientifique. Pour contrer cette tendance, R. Mocikat invite à encourager la pluralité linguistique parmi les scientifiques de son pays, et au-delà.

Le texte de H. Wismann «La diversité linguistique et la conceptualisation scientifique, le cas des sciences humaines» se démarque du reste des contributions par son approche fondamentalement épistémologique. À partir de la thèse de Heinrich Rickert, philosophe néo-kantien de la fin du XIXe siècle, selon laquelle il existe deux intérêts majeurs dans la structuration de la science, le premier consistant à subsumer les cas particuliers sous des règles générales et le deuxième à ne s'intéresser qu'à des cas particuliers, H. Wismann explore, en quelques pages, l'usage de la notion de champ dans la sociologie de Bourdieu et le cheminement du concept d'individu. Cette exploration sert, au final, à démontrer que les idées qui viennent aux chercheurs sont tributaires de leur capacité à déplacer les habitudes d'utiliser tel ou tel terme dans tel ou tel sens, ce qui n'est évidemment possible que dans le cas des langues de culture des scientifiques eux-mêmes.

Dans sa contribution intitulée «Pourquoi l'anglais ne peut que progresser en France», J. C. Herreras démontre, preuves à l'appui, (différentes enquêtes réalisées par la Commission européenne de 2008 à 2012) à quel point la situation actuelle repose sur deux idées aussi fausses l'une que l'autre; la première se résumant à «tout le monde parle anglais» et servant à justifier la politique linguistique menée au sein du système éducatif et la deuxième, «on ne peut rien faire», justifiant le fait de ne rien changer. L'auteur insiste sur le fait que la France, en tant que pivot de la francophonie, doit servir d'exemple à d'autres pays francophones et s'appuyer sur eux pour mettre en place une politique linguistique de non-discrimination face à l'anglicisation générale.

Dans son texte consacré à l'anglicisation des formations supérieures et de la recherche en France, P. Frath examine à la fois ses causes et ses conséquences, en concluant sur quelques pistes d'ac- 
Compte-rendu A. Prohić tion en vue d'un inversement de situation. Démontrant que ce sont, avant tout, les grandes écoles, ceux de commerce notamment, qui sont le fer de lance de cette anglicisation, l'auteur prend comme exemple le cas de l'Allemagne pour expliquer comment une telle politique mène à la perte d'un tas de domaines scientifiques, en l'occurrence de la terminologie nécessaire pour les soutenir et en assurer la créativité et la mémoire tout en baissant le niveau d'enseignement général et menant à une perte d'influence irréversible.

Pour expliquer les causes de cette anglicisation exacerbée, P. Frath s'appuie sur un vécu personnel et compare l'actuelle position dominante de l'anglais à celle de la langue française en Alsace après la Seconde Guerre Mondiale, où le sentiment de honte et un désir de soumission inconscients se sont emparés des locuteurs de l'alsacien au profit de la langue nationale. Il propose de réfléchir à une politique linguistique qui s'organiserait autour de quatre axes promouvant le français: développement de la production intellectuelle et artistique, augmentation de la visibilité à travers les médias, politique d'accords multilatéraux, coopération avec les pays qui souhaitent développer une éducation en français.

En partant de l'opposition nécessairement synchronique entre langues de service (dans le cas européen, l'anglais) et langues de culture traditionnelles (telles que, dans le contexte européen, le grec, le français, l'allemand, l'italien et d'autres), P. Krimpas consacre sa contribution à la valeur historique et, à travers la création terminologique, à la pratique du grec moderne dans les milieux scientifiques. Il dresse le constat d'une anglicisation du discours grec, notamment dans les domaines technologiques et scientifiques, mais cite aussi une myriade d'institutions nationales qui soutiennent la terminologisation hellénique et une législation qui protège encore suffisamment la langue grecque, ne serait-ce que dans l'enseignement supérieur.

Le dernier article de l'ouvrage est signé P. Blanchet qui, lui, propose une synthèse de ses précédents travaux portant sur le rapport français/anglais dans l'élaboration et la diffusion de la recherche scientifique, notamment celle des sciences humaines et sociales. Pour P. Blanchet, d'orientation bourdieusienne, ce rapport est comparable à celui existant entre français du Nord et français du Sud, dans la mesure où l'apprentissage en français, décontextualisé de l'environnement social et des besoins éducatifs des étudiants des pays du Sud, rappelle l'hégémonie des acteurs anglophones sur l'activité scientifique internationale. La problé- 


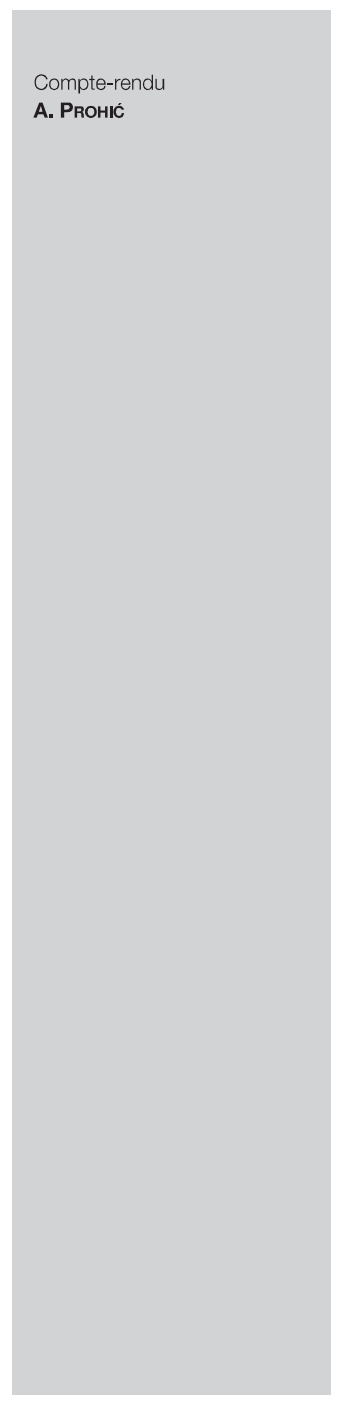

matique est présente aussi bien en aval qu'en amont. En aval sur le plan des ressources humaines, institutionnelles et documentaires, qui ont toutes un impact sur les choix de langues des chercheurs et les possibilités méthodologiques, notamment dans le domaine des sciences humaines et sociales. En amont, au niveau de la diffusion des connaissances, plus particulièrement dans le domaine de l'édition et de l'accès aux publications scientifiques.

Avec le chapitre «Lectures», l'ouvrage se termine par quelques réflexions de $\mathrm{P}$. Frath sur le livre collectif De l'idéologie monolingue à la doxa plurilingue: regards pluridisciplinaires $(\mathrm{H}$. Adami et $\mathrm{V}$. André (éds)., Peter Lang, Bern, 2015) faisant référence au livre de B. Maurer (1) critique ardent de la politique linguistique du Conseil de l'Europe.

Au-delà du compte-rendu des différents textes composant l'ouvrage en question, P. Frath élabore une analyse des argumentaires des auteur(e)s, ce qui lui permet de développer de manière précise une classification des langues et des principes selon laquelle leur gestion devrait s'articuler pour le maintien du plurilinguisme face à la prédominance de l'anglais.

À l'heure où le gouvernement français s'apprête à mettre en place une trentaine de mesures pour renforcer la place et le rôle de la langue française et du plurilinguisme dans le monde, il reste à voir jusqu'à quel point les souhaits exprimés dans cet ouvrage pourront être exaucés.

\section{NOTE}

(1) Bruno Maurer, Enseignement des langues et construction européenne - Le plurilinguisme, nouvelle idéologie dominante, Éditions des Archives Contemporaines, Paris, 2011. 